\title{
Procesado y propiedades de impacto de materiales compuestos laminados
} de base hierro

\author{
F. Carreño*, M. Pozuelo*, J. Chao* y Ó.A. Ruano*
}

Resumen

\begin{abstract}
Se ha procesado un material compuesto laminado de base hierro, de 7 capas alternas de dos aceros diferentes (cuatro capas de acero de ultra-alto contenido en carbono, AUAC, y tres de acero suave, AS), mediante unión por laminación y se ha estudiado su microestructura y propiedades de impacto. Se eligieron los parámetros adecuados de temperatura y de reducción de espesor para obtener una microestructura más fina que la de los aceros constituyentes originales, lo que induce una mejora en las propiedades mecánicas. Los valores de energía absorbida en el ensayo Charpy, tanto en la dirección "en paralelo" como "en serie", han mejorado sustancialmente el valor asociado al AUAC monolítico. Además, el valor obtenido en la dirección "en serie" es superior al del AS. Se observa que la delaminación, que depende de la unión de las intercaras, juega un papel importante en la deflexión de las fisuras, absorbiendo energía e imponiendo sucesivas nucleaciones de grietas en las siguientes capas.
\end{abstract}

Palabras clave Procesado. Materiales compuestos laminados. Microestructura. Impacto. Resiliencia.

\section{Processing and impact properties of steel based laminated composites}

\begin{abstract}
A seven layers steel based laminated composite (four ultra-high carbon steel, UHCS, layers and three mild steel, MS, layers) has been processed by rolling bonding and its microstructure and impact properties have been studied. Suitable parameters of temperature and thickness reduction were selected to obtain a finer microstructure relative to the original material components. This finer microstructure induces improved mechanical properties. Charpy impact tests values in both "crack arrester" and "crack divider" orientations improve the values of the UHCS constituent material. Furthermore, the crack arrester orientation value exceed that of the MS material. The delamination, which is controlled by interface bonding, plays a key role deflecting the crack, absorbing energy and imposing the nucleation of new cracks in the next material layer.
\end{abstract}

Keywords Processing. Laminated composites materials. Microstructure. Impact. Resilience.

\section{INTRODUCCIÓN}

Los materiales compuestos laminados constan de diferentes constituyentes separados alternativamente por intercaras discretas. Estas intercaras son las responsables de las interesantes propiedades mecánicas de los compuestos laminados, que mejoran las de los materiales constituyentes. Tal es el caso de las propiedades de fractura e impacto, donde los mecanismos extrínsecos de fractura operan alterando la propagación de las grietas debido a la presencia de las intercaras.
Por el contrario, se espera que la resistencia mecánica del compuesto laminado sea la media de la de los componentes, aunque mediante un procesado adecuado, por ejemplo, vía "unión por laminación"(roll bonding), puede mejorarse la resistencia de cada uno de los componentes a través de un afino de su microestructura.

La ductilidad del compuesto laminado también se espera más elevada que la media de la de los constituyentes. Una intercara fuertemente unida, llevará asociada mayor ductilidad ${ }^{[1-3]}$. Sin embargo, las propiedades de impacto disminuirán ya que en

(*) Departamento de Metalurgia Física. Centro Nacional de Investigaciones Metalúrgicas (CENIM). C.S.I.C. Avda. Gregorio del Amo, 8. 28040 Madrid (España). 
una intercara fuertemente unida la delaminación ocurrirá más difícilmente ${ }^{[4]}$. Por ello, se consigue un balance adecuado entre ductilidad y tenacidad controlando la unión de las intercaras.

Se pueden elegir diferentes caminos de procesado para la elaboración de los compuestos laminados, por ejemplo unión por compresión (press bonding) y unión por laminación (roll bonding $)^{[3-5]}$. Se ha elegido la unión por laminación por presentar una serie de ventajas, respecto a la unión por compresión, debidas a la deformación plástica inducida en el material, lo cual mejora la unión de las intercaras y mejora también la microestructura, afinando el tamaño de grano, globulizando carburos, etc.

En este trabajo se estudia la producción, microestructura y resiliencia de un material compuesto laminado, de base hierro, procesado mediante unión por laminación.

\section{PARTE EXPERIMENTAL}

\subsection{Material y procedimiento experimental}

Se emplearon dos aceros diferentes como materiales constituyentes del compuesto laminado: un acero suave de bajo contenido en carbono (AS) de composición 0,035 \% C, 0,28 \% Mn, 0,03 \% Si y un acero de ultra-alto contenido en carbono (AUAC) de composición 1,55\% C, 0,49\% Mn, $0,05 \% \mathrm{Si}, 1,55 \% \mathrm{Cr}, 0,17 \% \mathrm{Al}$ (\% en masa). Ambos aceros habían sido laminados, obteniéndose chapas de 4 y $4,5 \mathrm{~mm}$ de espesor para el AS y el AUAC, respectivamente.

Se cortaron y mecanizaron cuadrados de $6 \times 6$ $\mathrm{cm}$ de los dos aceros, presentando sus caras lisas y limpias. Siete de estos cuadrados fueron apilados formando la secuencia AUAC-AS-...-AUAC. Después fueron soldados herméticamente con TIG en sus bordes para evitar la penetración de oxígeno y la delaminación durante el proceso de laminación a alta temperatura. El espesor inicial del tocho fue de $29,5 \mathrm{~mm}$, que expuesto durante aproximadamente $1 \mathrm{~h}$ a $650^{\circ} \mathrm{C}$ y, mediante 6 series de 3 pasadas con una reducción aproximada de $5 \%$ en cada paso se llegó a un espesor final de 10,5 $\mathrm{mm}$. Por tanto, la reducción total fue de aproximadamente $3: 1$. Durante las diferentes series, el material fue introducido $5 \mathrm{~min}$ en el horno, a la misma temperatura.

La microestructura de cada uno de los constituyentes y del material compuesto laminado fue observada mediante el microscopio electrónico de barrido (MEB). Las piezas seleccionadas fueron cortadas, pulidas y atacadas con Nital al $2 \%$ para revelar la microestructura de ambos aceros y la calidad de las intercaras.

Se mecanizaron probetas tipo Charpy con entalla en $\mathrm{V}$ de $2 \mathrm{~mm}$, tanto de los materiales constituyentes como del compuesto laminado y se ensayaron a impacto con un péndulo de $30 \mathrm{kgm}$ para medir su resiliencia. Las dimensiones de las probetas del compuesto laminado fueron de $10 \times 7,4 \times 55 \mathrm{~mm}$. Tres de ellas fueron mecanizadas en la orientación "en serie" y otras tres "en paralelo" como muestra la figura 1 . Las dimensiones de las probetas AS fueron de $10 \times 3,8 \times 55 \mathrm{~mm}$ y de $10 \times 4,6 \times 55 \mathrm{~mm}$ las de AUAC. Las superficies de fractura de las muestras ensayadas a impacto fueron observadas en el MEB.

\section{RESULTADOS Y DISCUSION}

La microestructura inicial de los materiales originales sufrió cambios como consecuencia del procesado. Estos cambios se pudieron observar en el MEB al igual que las superficies de fractura de las probetas Charpy ensayadas.

\subsection{Microestructura inicial}

Las figuras 2 a) y 2 b) muestran la microestructura inicial del AUAC y AS respectivamente. El

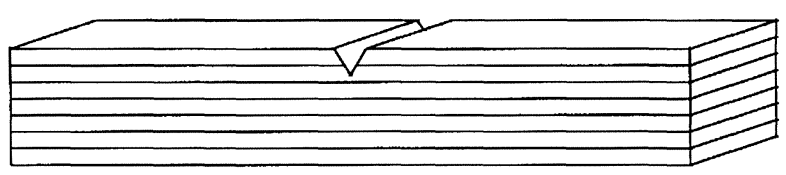

a)

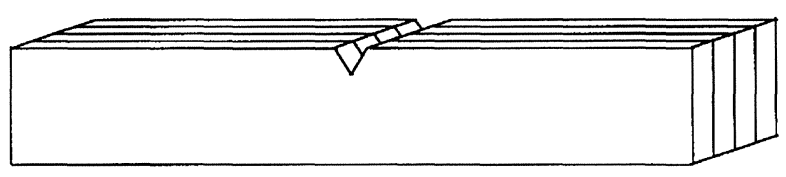

b)

Figura 1. Orientaciones en el ensayo de impacto Charpy: a) "en serie" y b) "en paralelo".

Figure 1. Orientations used for the impact Charpy tests: al "crack arrester" and b) "crack divider". 

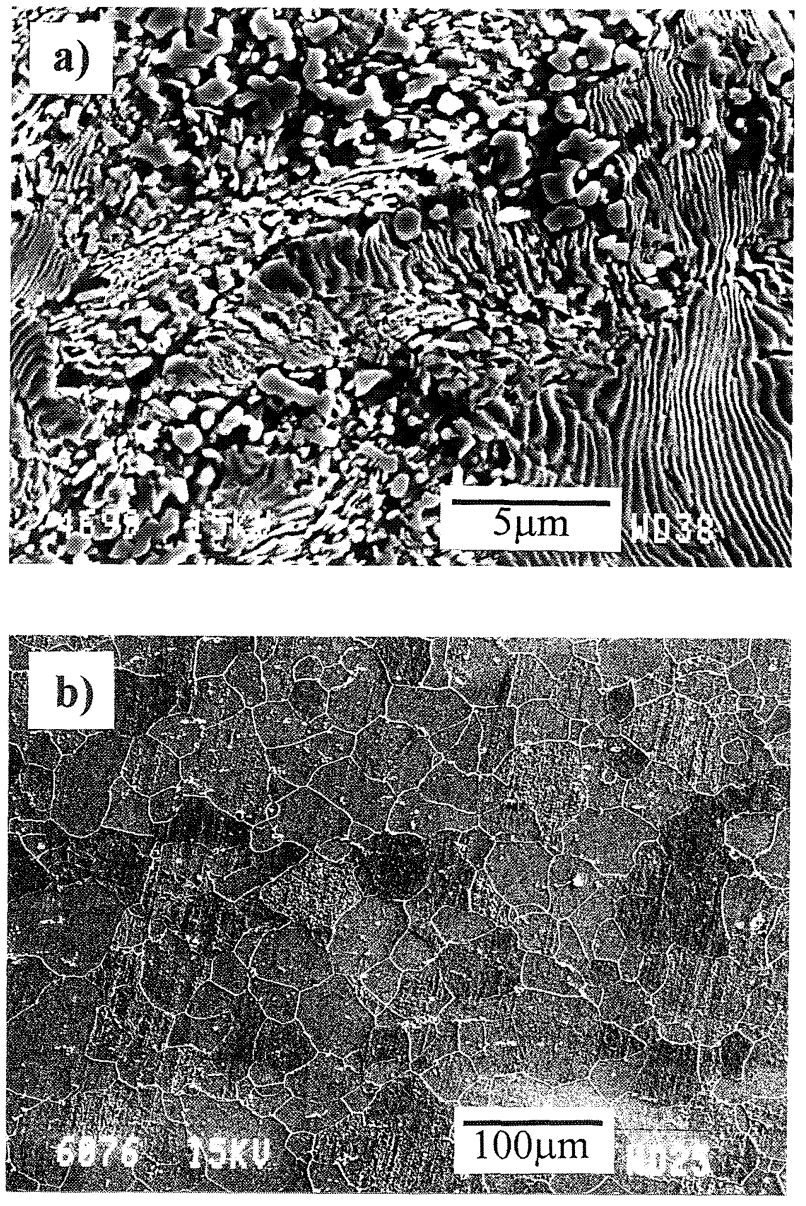

Figura 2. Microestructuras iniciales observadas en el MEB: a) AUAC y b) AS.

Figure 2. Initial microstructures observed in the SEM: a) UHCS and b) MS.

AUAC presenta una fracción de volumen de carburos de hierro, cementita, muy elevada. Esta cementita se encuentra en su mayoría globulizada con unas dimensiones de alrededor de $1 \mu \mathrm{m}$, aunque también se observan grandes colonias perlíticas con carburos de alrededor de $10 \mu \mathrm{m}$. Por otro lado, en el AS no se observa una cantidad apreciable de carburos de $\mathrm{Fe}$, ni otras fases en la matriz de Fe y su tamaño de grano, $\mathrm{L}$, es de $50 \mu \mathrm{m}$, aproximadamente.

\subsection{Microestructura del compuesto laminado}

Observaciones en el MEB (Fig. 3) revelan cambios microestructurales en los materiales constituyentes una vez laminados a $650^{\circ} \mathrm{C}$. También se ha determinado el estado y calidad de las intercaras.

La micrografía de la figura 3, muestra que el AUAC presenta una microestructura más fina que la de partida. No hay zonas perlíticas visibles y los

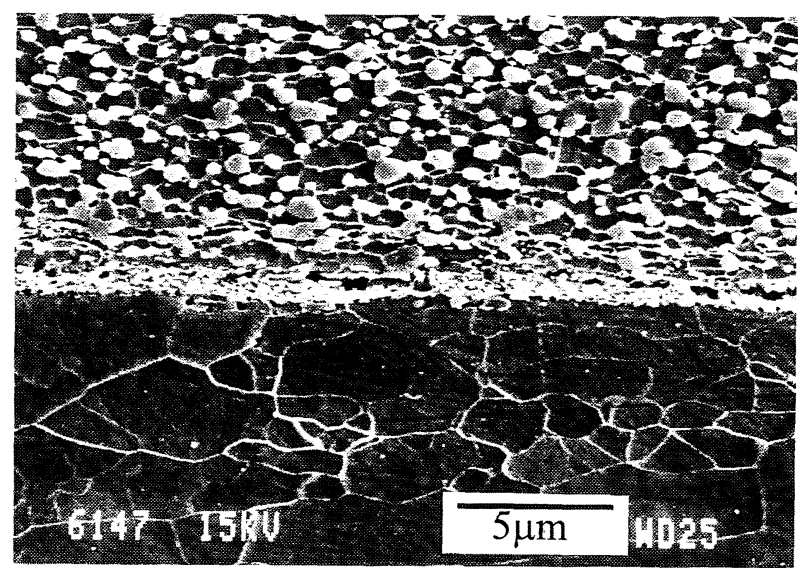

Figura 3. Micrografía MEB del material compuesto laminado de base Fe.

Figure 3. SEM micrograph of the steel based laminated composite material.

grandes carburos que aparecían en un principio se han ido transformando en pequeños y redondeados carburos, homogéneamente distribuidos en la matriz ferrítica. Estos pequeños carburos globulizados son ahora, incluso, inferiores a $1 \mu \mathrm{m}$ de diámetro. Por tanto, el procesado de fabricación del laminado ha mejorado sustancialmente la microestructura del AUAC, volviéndola más fina, globulizada y homogénea. Esta microestructura presenta ventajas frente a la que se tenía en un principio.

En cuanto al AS, se puede observar la micrografía de la figura $3^{[6 \text { y } 7]}$ tamaños de grano de alrededor de $10 \mu \mathrm{m}$, o incluso tan pequeños como de $2 \mu \mathrm{m}$ cerca de la intercara. Una reducción de 3:1 en el proceso de laminación conlleva un afinamiento de grano considerable en el AS.

Se observa también una intercara muy definida entre el AS y el AUAC, sin percibirse interdifusión de carbono entre las capas de los distintos aceros.

\subsection{Ensayos de impacto}

Se llevaron a cabo dos ensayos de impacto con probetas Charpy entalladas en $\mathrm{V}$ para cada uno de los materiales constituyentes y otros tres para el compuesto laminado en cada una de las dos orientaciones, "en serie" y "en paralelo". La tabla I muestra los datos obtenidos en estos ensayos.

El compuesto laminado, en cualquiera de las dos orientaciones, da un valor Charpy más alto que el obtenido para el AUAC originario. Para la orientación "en paralelo" el aumento es de 6 veces y para la orientación "en serie" de 35 veces, superior. 
Tabla I. Valores de resiliencia obtenidos en el ensayo de impacto Charpy

Table I. Resilience values from impact Charpy tests

\begin{tabular}{lc}
\hline \multicolumn{1}{c}{ Material } & Resiliencia $\left(\mathrm{kgm} / \mathrm{cm}^{2}\right)$ \\
\hline AS & 9,7 \\
AUAC & 0,3 \\
Laminado "en serie" & 10,4 \\
Laminado "en paralelo" & 1,9 \\
\hline
\end{tabular}

Además, el valor de la resiliencia del compuesto laminado en la orientación "en serie" también supera al obtenido para el AS. Sin embargo, el valor que se obtiene en la orientación "en paralelo" no supera al del AS; en realidad, es más bajo que el esperado de la regla de las mezclas. Este valor esperado es de $4,3 \mathrm{kgm} / \mathrm{cm}^{2}$ teniendo en cuenta las fracciones de volumen de los materiales constituyentes.

El hecho de que el valor de resiliencia del laminado "en paralelo" sea menor que la mitad del valor esperado, se podría atribuir a que la fractura del compuesto está dominada por la fractura del material más débil, es decir, por el AUAC, lo cual estaría de acuerdo con la hipótesis de fallo del eslabón más débil. En la orientación "en paralelo" la grieta se propaga fácilmente a través del espesor de la probeta según un plano. Por otra parte, el valor de resiliencia indica una unión fuerte entre las láminas, puesto que el AUAC induce fragilidad en el AS. Sin embargo, como ya se dijo anteriormente, el valor de resiliencia es considerablemente mayor que el del AUAC.

La estimación del valor de resiliencia en la orientación "en serie" es algo más compleja, dado que no todas las capas están sometidas al mismo estado tensional que se produce alrededor de la entalla. Las delaminaciones que pudieran ocurrir transformarían una probeta agrietada en una sin agrietar, implicando un gran consumo de energía mecánica, como sugiere la figura 4. Esta figura muestra una probeta ensayada en la orientación "en serie". La propagación de la grieta es más difícil en esta orientación debido a las sucesivas discontinuidades que provocan las intercaras de los materiales. En estas intercaras tienen lugar mecanismos extrínsecos de fractura, tales como delaminación y nucleación de nuevas grietas, que dificultan la propagación fácil de las mismas en el siguiente material en ambas orientaciones ${ }^{[4]}$.

Durante la delaminación, que depende de la unión entre intercaras, se produce una absorción

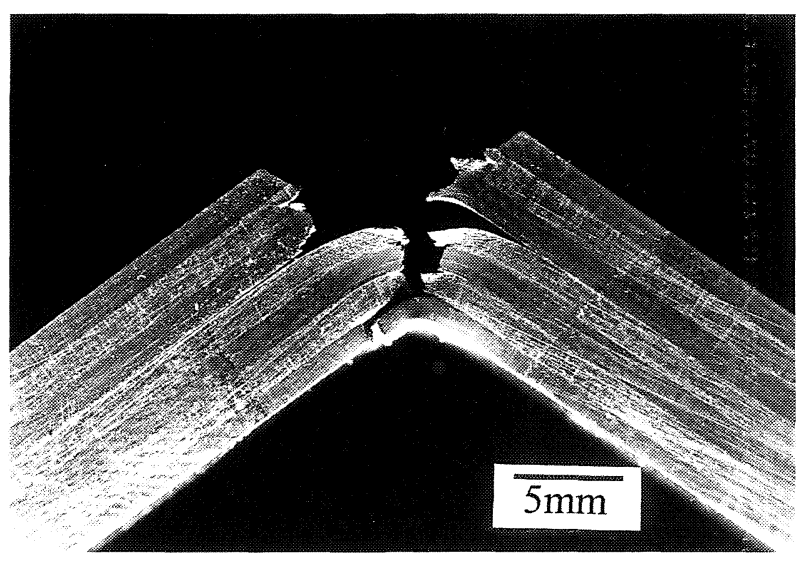

Figura 4. Probeta Charpy del material compuesto laminado ensayada en la orientación "en serie".

Figure 4. Charpy notched impact sample of the laminated composite material tested in the "crack arrester" orientation.

de energía. Adicionalmente, una vez producida la delaminación de una intercara, debe nuclearse una nueva grieta para propagarse en el siguiente material $^{[8}$ y 9]. La delaminación, por tanto, depende de la calidad de la unión de las intercaras entre materiales constituyentes. La unión de las intercaras se puede fortalecer, aún más, en el presente material compuesto laminado mediante tratamientos térmicos, dado que no se observó interdifusión de carbono entre los aceros constituyentes ${ }^{[5]}$. Sin embargo, si la unión fuese muy fuerte, el comportamiento del material compuesto frente al impacto podría ser cercano al del más frágil. En este caso la grieta se propagaría rápidamente en el material más frágil, el AUAC, y no se vería frenada por la intercara, induciéndose fácilmente la nucleación y la propagación en el material siguiente, el AS. Entonces, los valores de la energía de impacto esperados serían menores que los obtenidos experimentalmente. Por el contrario, si la unión fuese muy débil, no se absorbería ninguna energía al delaminar, aunque se gastaría energía para nuclear las grietas en las sucesivas láminas en la orientación "en serie". En la orientación "en paralelo" el valor esperado de resiliencia sería la media de la de los aceros constituyentes. Por lo tanto, para optimizar la resistencia a la fractura y al impacto de los materiales compuestos laminados se ha de buscar un compromiso entre unión fuerte y débil de las láminas que dé lugar a un mayor valor de energía absorbida.

En resumen, se puede establecer que las mejores propiedades de impacto se lograrán cuando la unión de las intercaras mantenga un compromiso 
entre unión fuerte y débil que aumente la absorción de energía del material compuesto laminado. Esta mejora en la resistencia al impacto se puede lograr, entre otros factores, controlando la presión y temperatura durante la unión por laminación. Adicionalmente, se espera que las propiedades de impacto se mejoren con un aumento del número de capas y una reducción de sus espesores.

\section{CONCLUSIONES}

Del estudio del procesado y ensayo a impacto de un material compuesto laminado de 7 capas de aceros (4 de acero AUAC y tres de acero AS) se concluye:

- La microestructura inicial del acero suave, AS, caracterizada por un tamaño de grano de $50 \mu \mathrm{m}$, se transformó, una vez sometido el material al proceso de laminación, en una microestructura más fina con un tamaño de grano incluso por debajo de $10 \mu \mathrm{m}$.

- El proceso de laminación modificó, también, la microestructura inicial del AUAC, caracterizada por una alta fracción de volumen de carburos de Fe en colonias de perlita y perlita parcialmente globulizada de, aproximadamente, $2 \mu \mathrm{m}$ de diámetro. Esta modificación de la microestructura consistió en la eliminación de las colonias de perlita y la globulización de los carburos a un tamaño de alrededor de $1 \mu \mathrm{m}$.

- Las intercaras del compuesto laminado permanecieron abruptas y no se observó difusión aparente de carbono entre las capas.

- La resiliencia del compuesto laminado es más elevada que la del AUAC tanto en la orientación "en serie" como "en paralelo". Sin embargo, la resiliencia sólo es más elevada que la del AS en la orientación "en serie". Esto pone de relieve la operación de mecanismos extrínsecos de fractura, tales como la delaminación y nucleación de grietas.

- La orientación "en paralelo" muestra valores de impacto más bajos que los esperados de la regla de las mezclas, implicando una unión de intercara fuerte. Una unión más débil sería más efectiva en promover mecanismos de fractura extrínsecos, no solamente en la dirección "en serie" sino también "en paralelo".

- Los mecanismos extrínsecos de fractura que operan en el material compuesto laminado es- tán controlados por la unión de las intercaras. Esta unión juega un papel clave en la desviación de la grieta, absorbiendo energía e imponiendo la nucleación de nuevas grietas en la siguiente capa.

\section{Agradecimientos}

Los autores desean expresar su agradecimiento a la CICYT por la financiación recibida a través del proyecto PETRI 95-0091-OP. F. Carreño agradece a la Comunidad Autónoma de Madrid la concesión de una beca postdoctoral. También se agradece a D. Luis del Real Alarcón por llevar a cabo la soldadura de los tochos antes de ser laminados, a D. Fernando Federico González Rodríguez por su colaboración en la laminación en caliente, a D. Antonio Viñuales por la mecanización de los aceros, y a D. Angel Fernández Ontiveros por su asistencia durante los ensayos Charpy. Se aprecian muy sinceramente las discusiones mantenidas con el Prof. O.D. Sherby.

\section{REFERENCIAS}

[1] C.K. Syn, D.R. Lesuer, K.L. Cadwell, O.D. Sherby y K.R. BROWN, Developments in ceramic and metal-matrix composites, Ed. Kamleshwar Upadhya, The Minerals, Metals \& Materials Society, 1991, pp. 311.

[2] C.K. Syn, D.R. Lesuer, J. Wolfenstine y O.D. Sherby, Metall. Trans. A 24 (1993) 1647.

[3] C.K. Syn, D.R. Lesuer y O.D. Sherby, Mater. Sci. Eng. A 206 (1996) 201.

[4] D.R. Lesuer, C.K. Syn, O.D. Sherby, J. Wadsworth, J.J. LEWANDOWSKI y W.H. Hunt, JR., Int. Mater. Rev. 41 (1996) 169.

[5] D.W. Kum, T. Oyama, O.A. Ruano y O.D. Sherby, Metall. Trans. A 17 (1986) 1517.

[6] D.R. Lesuer, C.K. Syn, A. Goldberg, J. Wadsworth y O.D. Sherby, J. Miner. Mater Soc. 45 (1993) 40.

[7] D. R. Lesuer, C. K. Syn y O. D. Sherby, Acta Metall. Mater. 43 (1995) 3827.

[8] D.R. Lesuer, C. K. Syn, R. Riddle y O.D. Sherby, Editado por J.J. Lewandowsky y W.H. Hunt, Jr. The Minerals, Metals Ë Materials Society 1995, pp. 93.

[9] D.R. Bloyer, K.T. Venkateswara RAO y R.O. Ritchie, Metall. Mater. Trans. A 29 (1998) 2483.

[10] G. Banu-PraKash-Babu y R.K. Dube, ISIJ Int. 36 (1996) 1184. 